\title{
Transfer Functions of Laminar Premixed Flames Subjected to Forcing by Acoustic Waves, AC Electric Fields, and Non-thermal Plasma Discharges
}

\author{
Deanna A. Lacoste ${ }^{\mathrm{a}}$, Yuan Xiong ${ }^{\mathrm{a}}$, Jonas P. Moeck ${ }^{\mathrm{b}}$, Suk Ho Chung ${ }^{\mathrm{a}}$, \\ William L. Roberts ${ }^{\mathrm{a}}$, Min Suk Cha ${ }^{\mathrm{a}}$ \\ ${ }^{a}$ King Abdullah University of Science and Technology, Clean Combustion Research \\ Center, Thuwal 23955-6900, Saudi Arabia \\ ${ }^{b}$ Technische Universität Berlin, Institut für Strömungsmechanik und Technische Akustik, \\ 10623 Berlin, Germany
}

\begin{abstract}
The responses of laminar methane-air flames to forcing by acoustic waves, AC electric fields, and nanosecond repetitively pulsed (NRP) glow discharges are reported here. The experimental setup consists of an axisymmetric burner with a nozzle made from a quartz tube. Three different flame geometries have been studied: conical, M-shaped and V-shaped flames. A central stainless steel rod is used as a cathode for the electric field and plasma excitations. The acoustic forcing is obtained with a loudspeaker located at the bottom part of the burner. For forcing by $\mathrm{AC}$ electric fields, a metallic grid is placed above the rod and connected to an AC power supply. Plasma forcing is obtained by applying high-voltage pulses of 10-ns duration applied at $10 \mathrm{kHz}$, between the rod and an annular stainless steel ring, placed at the outlet of the quartz tube. The chemiluminescence of $\mathrm{CH}^{*}$ is used to determine the heat release rate fluctuations. For forcing by acoustic waves and plasma, the geometry of the flame plays a key role in the response of the combustion, while the
\end{abstract}


flame shape does not affect the response of the combustion to electric field forcing. The flame response to acoustic forcing of about $10 \%$ of the incoming flow is similar to those obtained in the literature. The flames are found to be responsive to an $\mathrm{AC}$ electric field across the whole range of frequencies studied. A forcing mechanism, based on the generation of ionic wind, is proposed. The gain of the transfer function obtained for plasma forcing is found to be up to 5 times higher than for acoustic forcing. A possible mechanism of plasma forcing is introduced.

Keywords: plasma-assisted combustion, thermoacoustic instabilities, nanosecond repetitively pulsed discharges, combustion dynamics

\section{Introduction}

Mitigation of thermoacoustic instabilities is a major challenge in the design of modern stationary gas turbines and aeroengines [1]. These instabilities are a dynamic phenomenon resulting from the interaction of combustion generated unsteady heat release rate (HRR) and acoustic modes of the combustion chamber [2]. They lead to high-amplitude oscillations of the pressure, the flow field, and the flame, and are therefore detrimental to industrial applications. Recently, several studies have shown that nanosecond repetitively pulsed (NRP) discharges [3-7], as well as an electric field [8-13] can significantly affect flame dynamics.

For example, it has been shown that thermoacoustic instabilities of a 50$\mathrm{kW}$ natural gas-air burner can be suppressed by a plasma actuation using NRP discharges with an electrical power of $500 \mathrm{~W}$, i.e., about $1 \%$ of the thermal power released by the flame [4]. Similarly, Kim et al. [7] have shown 
that a pressure peak produced by thermoacoustic instability can be decreased by up to $25 \mathrm{~dB}$ with the help of nanosecond plasma discharges applied at $30 \mathrm{kHz}$ pulse repetition rate.

When a sub-critical voltage, i.e., lower than the breakdown voltage, is applied to a flame, a modulation of the combustion front caused by an electric field can be obtained, without electrical discharge. In this case, the flame modulation results mainly from a hydrodynamic effect instead of the heating and chemical effects present when applying plasma discharges. This electricfield-induced hydrodynamic effect, especially under an AC electric field, is receiving increased attention for both diffusion and premixed flames. For example, Xiong et al. [14] found that the Lorenz force associated with an $\mathrm{AC}$ electric field could trigger an inner toroidal vortex in the area close to the nozzle of a small diffusion flame. Drews et al. [15] explored the response of a premixed flame under AC electric fields and observed the creation of a steady flow pattern. The response of laminar premixed methane-air flames to steps of a DC electric field was investigated by Kuhl et al. [10]. They have shown that the response of the flame can be as fast as $3 \mathrm{~ms}$. This result was attributed to an ionic wind effect, with a maximum ionic wind of $1.6 \mathrm{~m} / \mathrm{s}$. The response of the flame to an AC electric field was investigated by GuerraGarcia et al. [12] with counterflow diffusion flames. The action of AC electric fields, in the frequency range of $2 \mathrm{~Hz}$ to $35 \mathrm{kHz}$, were also explained by ionic winds effects. To fully realize the AC-induced hydrodynamic effect for flame modulation, the flame response should first be characterized.

A common approach to characterize the response of a flame to acoustic forcing is based on the flame transfer function (FTF) formalism. This system- 
atic analysis of the response of a flame to a large range of forcing frequencies can lead to a better understanding of the forcing mechanisms [16-20]. In previous work, Lacoste et al. [5] and Volkov et al. [9] have shown that both NRP plasma discharges and DC electric fields can significantly affect the response of premixed flames to acoustic forcing. However, to the best of our knowledge, the transfer function of a flame to plasma or $\mathrm{AC}$ electric field forcing, in the absence of acoustic waves, has never been investigated.

Therefore, in this study the response of premixed flames to forcing by acoustic waves, AC electric fields, and non-thermal plasmas is investigated and compared, possible forcing mechanisms are then discussed.

\section{Experimental setup}

Depending on the forcing source, two slightly different experimental setups have been used. Figure 1 presents the burner, equipped for forcing by acoustic and electric fields (a) and for the plasma forcing (b).

\subsection{Laminar premixed burner}

An axisymmetric laminar burner, fed with a premixture of methane and air, has been used. The flows of air and methane, controlled by mass flowmeters, are injected at the lowest part of a $120-\mathrm{cm}$ long plenum. In order to minimize the boundary layer at the outlet of the injection tube, the flow passes through a nozzle, similar to the one used by Mazas et al. [21]. The contraction ratio is 86 , with a 7 -mm inner diameter injection tube, made from quartz. A stainless steel rod, $0.85-\mathrm{mm}$ in diameter, is centered inside the quartz tube and oversteps its outlet by $2 \mathrm{~mm}$. An equivalence ratio of 

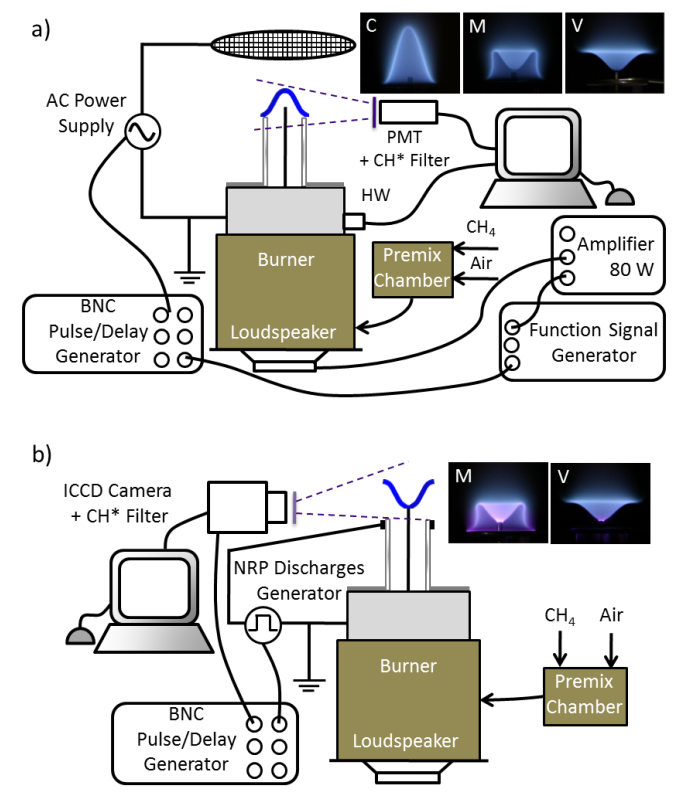

Figure 1: Experimental setup used for a) acoustic and AC electric field forcing with three flame geometries: conical (C), M-shaped (M), and V-shaped (V) flames, and b) plasma forcing by NRP glow discharges with two flame geometries: M-shaped (M) and V-shaped (V) flames. 
0.95 has been chosen in order to allow stabilization of different flame geometries. Depending on the location of the ignition source, the flame can thus be stabilized in three different shapes: a conical flame (C), an M-shape flame (M), and an inverted conical flame (V), (see Fig.1a). The flow rates of fuel and air are chosen to obtain a thermal power of $113 \mathrm{~W}$, corresponding to an axial velocity of the flow at the outlet of the quartz tube of about three times the laminar burning velocity for these conditions.

\subsection{Forcing systems}

The acoustic forcing source is secured by a loudspeaker (Monacor Number One SPH-115, $80 \mathrm{~W} / 8 \Omega, 55 \mathrm{~W}$ RMS), mounted at the bottom of the plenum. The loudspeaker is connected to an audio amplifier (OSC GX5 $500 \mathrm{~W}$ ) fed by a signal synthesizer (Tektronix AFG 3012B), with harmonic excitation. The resulting velocity perturbation is measured by a hot wire anemometer (Dantec probe 55P16), located inside the burner, $4 \mathrm{~cm}$ before the outlet of the injection tube. Previous work has shown that this distance between the velocity measurement region and the flame location does not introduce significant changes in the transfer function, especially for frequencies lower than $1 \mathrm{kHz}$ [22]. The forcing amplitude for the case considered here is $u^{\prime} / \bar{u}=$ $10 \%$, where $u^{\prime}$ indicates the amplitude of the perturbation and $\bar{u}$ is the mean velocity of the flow. Flame transfer functions have been determined with and without the presence of a grid, located $4 \mathrm{~cm}$ above the rod. The results obtained were identical. The transfer functions for acoustic forcing presented in this paper have been obtained in the presence of the grid. The forcing frequencies were varied from 8 to $450 \mathrm{~Hz}$.

The electric field forcing has been obtained by applying an AC high volt- 
age between the rod, connected to the ground, and the grid, located $4 \mathrm{~cm}$ above the rod, and connected to the high-voltage output of a power supply (Trek 30/20 A) (see Fig. 1a). The peak-to-peak voltage, measured with a high voltage probe (Tektronix P6015A), is fixed at $2.1 \mathrm{kV}$ and the averaged voltage is set at $1 \mathrm{~V}$. These values were chosen in order to easily calculate the associated flame transfer function (see Eq. 3) and to avoid corona discharges. The AC voltage frequencies were varied from 4 to $450 \mathrm{~Hz}$.

The forcing by non-thermal plasma has been obtained by NRP discharges, generated with a solid-state pulse generator (FID Technologies, FPG 10$30 \mathrm{NM} 10)$. High voltage pulses have $10-\mathrm{ns}$ duration, $10-\mathrm{kHz}$ repetition frequency, and 8-kV amplitude. The stainless steel rod, in the center of the burner, is connected to the ground, and serves as the cathode. The anode is a stainless steel ring located at the outlet of the 1-mm thick quartz tube (see Fig. 1b). The energy deposited by the plasma is determined from the amplitude of the voltage and current pulses, through the electrodes, measured with a high voltage probe (Tektronix P6015A) and a Pearson Coil (Model 6565). Measurements were made with the flame present. Voltage and current signals were first synchronized, then, the voltage and conduction current waveforms (obtained after subtracting the capacitive current form the total current), were multiplied and integrated to obtain the energy deposition per pulse [23]. The average power of the plasma was determined by multiplying the energy deposited per pulse by the repetition frequency of the discharge. In this study, the power is kept constant at $2 \mathrm{~W}$ for the M-flames, and at $1.7 \mathrm{~W}$ for the $\mathrm{V}$-flames. Investigations with C-flames were not possible because C-flames turned into M-flames as soon as the plasma forcing 
was applied. The current measurements also permit confirmation of the glow regime of the NRP discharges, i.e., low conduction current compared to the total current [24], and to assess that the plasma produced is non-thermal. Due to its highly non-linear behavior, the plasma cannot be modulated as a sine function. The forcing strategy will be detailed in section 3.1, after validation of several hypothesis.

\subsection{Diagnostics}

The spontaneous emission of excited methylidyne radicals $\left(\mathrm{CH}^{*}\right)$ is used to determine the HRR fluctuations of the flames. This technique has been validated for perfectly premixed flames [25, 26], and it has been extended here, according to previous studies $[9,5]$, for the determination of HRR fluctuations of flames subjected to electric field and plasma forcing. However, in order to verify that the $\mathrm{CH}^{*}$ emission was not affected by the nature of the forcing, optical emission spectroscopy measurements were undertaken in the wavelength range of the light collection, i.e., between 410 and $450 \mathrm{~nm}$. Figure 2.a presents a comparison of the spectra obtained for the flame alone (blue), the flame with applied AC voltage (red), and the flame with NRP discharges (black). In the case of plasma forcing, as the light emission from the plasma (mainly due to $\mathrm{N}_{2}$ (C-B) transition, in the wavelength range of interest) cannot be spatially separated from the flame emission light (see Fig. 1b), the spectrum should be recorded between two plasma pulses. This requirement fixed the acquisition settings, kept identical for the three spectra presented in Fig. 2, i.e., with an integration time of $98 \mu \mathrm{s}$. The three spectra obtained are identical. Therefore, for similar acquisition conditions, the $\mathrm{CH}^{*}$ chemiluminescence of the flame is used to determine the HRR fluctuations, 
for the three types of forcing.
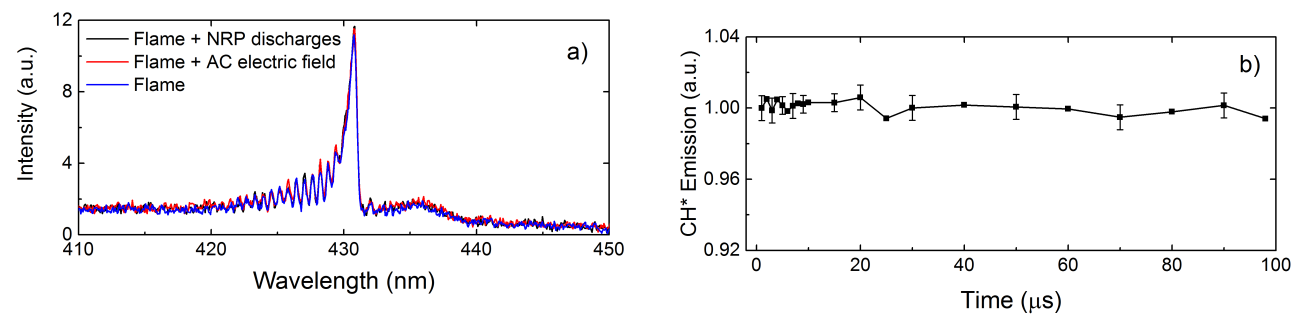

Figure 2: a) Comparison of flame spectrum (blue), spectrum of flame with an AC electric field (red), and flame emission between two pulses of NRP glow discharges (black). b) Temporal evolution of the global $\mathrm{CH}^{*}$ emission of an M-flame between two pulses of plasma.

For forcing by acoustic waves and AC electric field, a photomultiplier tube (PMT) (Hamamatsu H10721) equipped with a 10-nm bandpass filter (CVI Melles Griot F10-430.0-4-50.0M), centered at $430 \mathrm{~nm}$, was used. The location of the PMT has been chosen in order to collect the emission light from the entire combustion area, independent of the flame shapes.

For forcing by NRP discharges, it was not possible to collect the $\mathrm{CH}^{*}$ emission from the flame by the same technique because the PMT was not gated. Indeed, the light emission from the plasma pulses, in the same spectral range, would have been equally collected. Therefore, the measurements of $\mathrm{CH}^{*}$ chemiluminescence have been made with a $1024 \times 1024$-pixel intensifiedCCD camera (Princeton Instruments, PIMAX3) equipped with the same band pass filter used with the PMT. Thus, the signal collection has been gated and the light emission of the flame, between the plasma pulses, has been recorded. A delay generator (Berkeley Nucleonics, BNC Model 575) synchronized the FID pulse generator and the optical device to provide 
time-resolved information. For each forcing frequency studied, 16 images, phase-locked with the forcing signal, have been recorded, each image being an average of 200 events. An example of the flame motion generated by a plasma forcing at $64 \mathrm{~Hz}$ is presented in a movie, realized from the $16 \mathrm{CH}^{*}$ chemiluminescence images, see Fig.S1 of the supplemental material. After subtraction of the background, each image has been spatially averaged to obtain the averaged $\mathrm{CH}^{*}$ chemiluminescence intensity of the whole combustion area. The temporal evolution of the HRR has then been determined and analyzed to obtain the FTF.

\section{Flame transfer functions}

\subsection{Definitions for different forcing types}

When a flame is subjected to velocity perturbations $u^{\prime}$, the transfer function is defined as the ratio of the relative HRR fluctuation and the relative velocity fluctuation in the frequency domain:

$$
F_{a c}(\omega)=\frac{\hat{\dot{Q}}(\omega) / \overline{\dot{Q}}}{\hat{u}(\omega) / \bar{u}}
$$

where $(\hat{\cdot})$ denotes the Fourier transform of a variable. Practically, for each forcing frequency, the temporal evolution of the forcing signal, the velocity, and the $\mathrm{CH}^{*}$ emission intensity, were recorded during $16 \mathrm{~s}$. The flame transfer function is then obtained as the ratio of the cross power spectral density between the forcing signal and the relative chemiluminescence intensity fluctuation and the cross power spectral density between the forcing signal and the relative velocity fluctuation. 
The FTF can also be written in terms of a gain $G_{a c}$ and a phase $\phi_{a c}$ :

$$
F_{a c}(\omega)=G_{a c}(\omega) \mathrm{e}^{\mathrm{i} \phi_{a c}(\omega)},
$$

where $G_{a c}$ reflects the level of the flame response, and $\phi_{a c}$ defines the phase lag between the HRR and the velocity fluctuations at the base of the flame.

For AC electric field forcing, a transfer function, $F_{E}$, can be defined analogous to the one used for acoustic forcing:

$$
F_{E}(\omega)=\frac{\hat{\dot{Q}}(\omega) / \bar{Q}}{\hat{V}(\omega) / \bar{V}}=G_{E}(\omega) \mathrm{e}^{\mathrm{i} \phi_{E}(\omega)},
$$

where $V$ is the applied voltage and $\bar{V}$ is a reference value $(1 \mathrm{~V})$. Here also, the FTF can be expressed in terms of a gain $G_{E}$ and a phase $\phi_{E}$. It is important to note that the denominator of this transfer function is independent of the average thermal power of the flame. This general definition does not a priori assume a forcing mechanism, keeping open the explanation of the electric field effect. However, this definition will not allow quantitative comparisons between the gains obtained for acoustic and electric field forcing.

As the plasma formation is a highly nonlinear function of the input voltage, harmonic single-frequency forcing cannot be realized by NRP glow discharges. However, for small forcing amplitudes, at which the flame responds linearly, a square-wave forcing can be used equally well. Assuming that the flame is only affected by the average electrical power deposited by the plasma, square-wave forcing is applied by modulating the high-frequency pulse train with a gate signal at the intended forcing frequency, from 16 to $450 \mathrm{~Hz}$. This assumption has been validated by measuring the temporal evolution of the $\mathrm{CH}^{*}$-chemiluminescence between two plasma pulses (see Fig. 2b). The duty 
cycle of the gate signal is set to $50 \%$. The amplitude of the fundamental frequency component of the square-wave signal is related to the square-wave amplitude by a factor of $\operatorname{sinc}(1 / 2)$, which follows from a Fourier expansion of the square-wave signal. This factor must be included in the evaluation of the transfer function to plasma forcing, when it is written in terms of the fundamental component of the HRR fluctuation and the square wave amplitude:

$$
F_{P}(\omega)=\frac{\hat{\dot{Q}}(\omega) / \overline{\dot{Q}}}{\operatorname{sinc}(1 / 2) \cdot P_{P}(\omega) / \overline{\dot{Q}}}=G_{P}(\omega) \mathrm{e}^{\mathrm{i} \phi_{P}(\omega)}
$$

where $P_{P}$ is the average plasma power deposited during half a period of the forcing. Similar to Eqs. 2 and 3, $G_{P}$ and $\phi_{P}$ are the gain and the phase of the transfer function $F_{P}$. It is possible to define the amplitude of the forcing as $P_{P} / \bar{Q}$. Therefore, quantitative comparisons between the gains obtained for plasma and acoustic forcing will be possible.

Figure 3.a presents an example of the three forcing signals for a frequency of $196 \mathrm{~Hz}$, and Fig. 3.b presents the corresponding normalized HRR response of the M-flame.

To compare the transfer functions for the different flame geometries with previous results from the literature, a reduced angular frequency $\omega^{*}$, as defined in Durox et al. [19], is introduced:

$$
\omega^{*}=\frac{\omega R}{S_{L} \cos (\alpha)}=\frac{\omega R}{S_{L}\left(1-\left(S_{L} / u_{b}\right)^{2}\right)^{1 / 2}},
$$

where $\omega$ is the angular frequency, $R$ a characteristic dimension of the flame base, $S_{L}$ the laminar burning velocity, $\alpha$ the half angle at the top of the flame and $u_{b}$ the mean bulk velocity of the flow. 

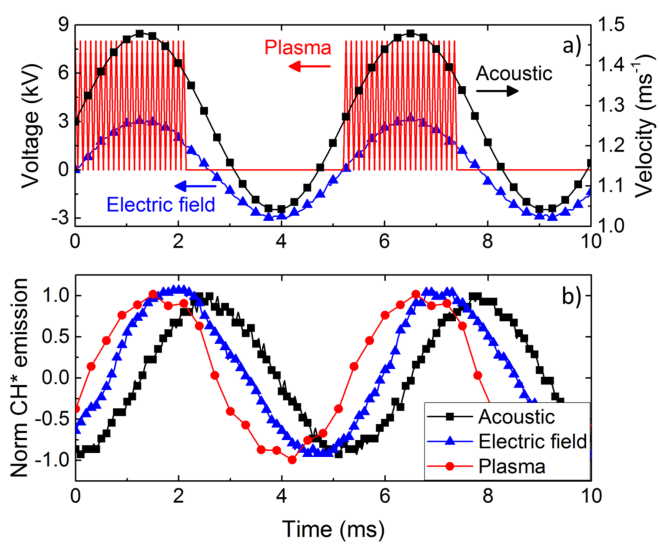

Figure 3: a) Example of forcing signals used for a forcing at $196 \mathrm{~Hz}$. b) Corresponding temporal evolution of the normalized $\mathrm{CH}^{*}$ emission of the M-flame.

To facilitate data interpretation in terms of dimensional values, the coefficient $K$, linking reduced frequency and real frequency, $f$, by $\omega^{*}=f / K$, is calculated for the three flame shapes, from the experimental conditions detailed in the previous section: $R=3.50 \mathrm{~mm}$ for the C-flames, $R=3.07 \mathrm{~mm}$ for the $\mathrm{V}$-flames, and $R=1.54 \mathrm{~mm}$ for the M-flames. $S_{L}$ is $0.35 \mathrm{~ms}^{-1}$ [21], and $u_{b}$, determined from velocity measurements at the outlet of the injection tube, is $1.26 \mathrm{~ms}^{-1}$. Thus, for the $\mathrm{C}-, \mathrm{V}-$, and M-flames $K$ is $15.0,17.1$, and $34.8 \mathrm{~s}^{-1}$, respectively. As all the experiments were conducted over the same range of forcing frequencies, i.e., from 4 to $450 \mathrm{~Hz}$, the data obtained for the M-flames extend to lower $\omega^{*}$ than for the two other flame shapes.

\subsection{Effect of the flame shape on the transfer functions}

The effect of the flame shape on the transfer functions obtained for the three types of forcing are presented in Figs. 4, 5 and 6. Taking experimental accuracy into account, uncertainty of the gains is about $10 \%$ of the nominal 
value, while phase uncertainty depends upon the forcing source. For acoustic forcing, uncertainty of the phase is a few percent of the nominal value. For electric field and plasma forcing, uncertainty of the phase is about $25 \%$ for reduced frequencies lower than 2.5 , and about $10 \%$ for higher frequencies. This appreciable uncertainty at low forcing frequencies is mainly due to the imperfect sinusoidal response of the flame.
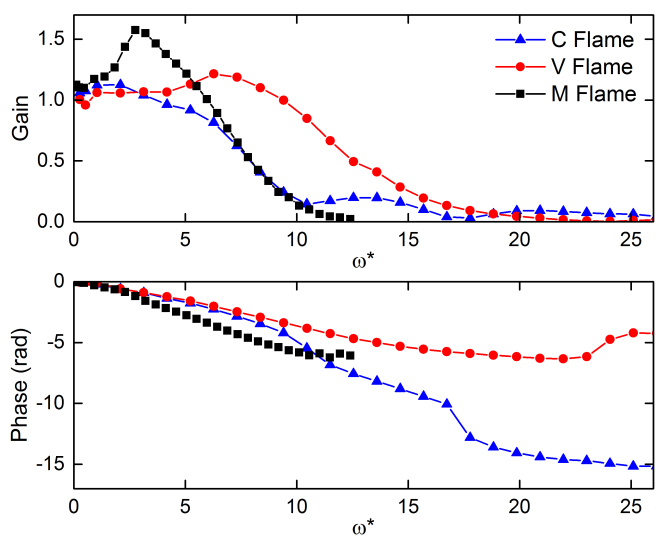

Figure 4: C-flame, V-flame and M-flame transfer functions to acoustic forcing (forcing amplitude 10\%).

Figure 4 presents transfer functions of C-, V- and M-flames for an acoustic forcing of $10 \%$ amplitude. The results are similar to those obtained by Durox et al. [19], confirming that flame shape plays a key role in the response of combustion to acoustic modulation of the flow.

Figure 5 presents transfer functions obtained for AC electric field forcing. First, it is important to note that the flame is responsive to electric field forcing over the entire range of reduced frequencies investigated. Second, conversely to acoustic forcing, the transfer functions of the three types of flames are relatively similar. The maximum gains are obtained for a reduced 

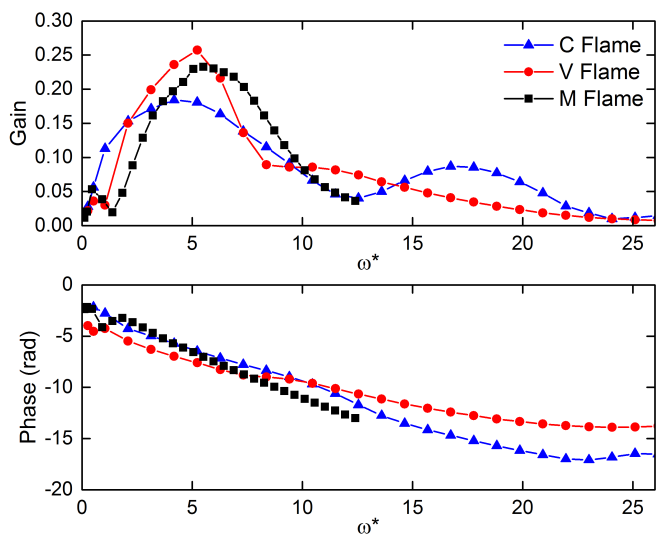

Figure 5: C-flame, V-flame and M-flame transfer functions to AC electric field forcing.

frequency of about 5, with slightly higher response amplitude for the $\mathrm{V}$ - and M-flames, compared to the C-flame. The phases are also very similar, with a linear evolution at similar slope. Consequently, it can be concluded that the flame response to AC electric field forcing is not significantly affected by its geometry; the forcing mechanisms might then be similar.
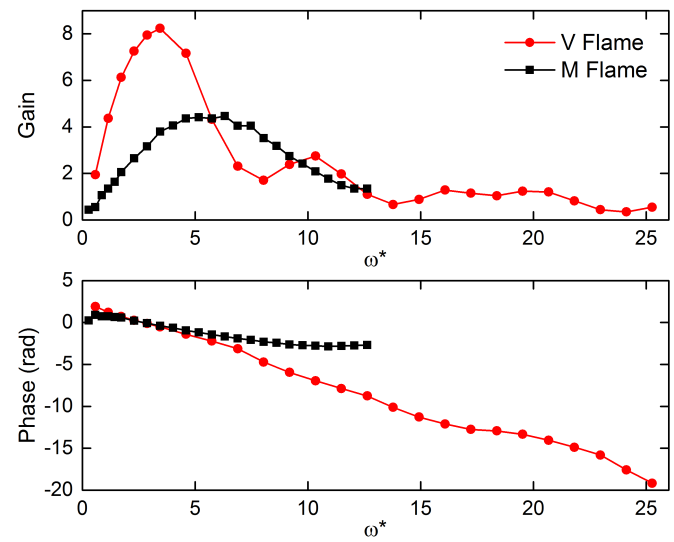

Figure 6: V-flame and M-flame transfer functions to non-thermal plasma generated by NRP glow discharges at $10 \mathrm{kHz}$ (forcing amplitude 1.8\%). 
Figure 6 presents the transfer functions obtained for plasma forcing, with an averaged plasma power of about $1.8 \%$ of the thermal power of the flame. The gain maximums are obtained for a reduced frequency of about 5, but the amplitude of the response of the V-flame is two times higher than the M-flame. In addition, even though the phases are both nearly linear, their slopes are very different. Thus, in the case of plasma forcing by NRP glow discharges, the shape of the flame seems to strongly influence the response of the combustion front.

\subsection{Comparison among the three types of forcing}

Gain maximums are sensitive to the forcing source. For electric field forcing, a maximum of 0.25 is obtained at $\omega^{*}=5$ (Fig. 5). This value is about six times lower than the maximum gain obtained for acoustic forcing (Fig. 4). However, taking into account the definition of the transfer functions (see Eqs. 3 and 4), this difference cannot be interpreted in terms of relative sensitivity of the flame to the forcing source.

Alternatively, for a plasma forcing of $1.8 \%$ amplitude, the maximum gain is more than three times higher than for an acoustic forcing of $10 \%$ amplitude. Even if lower acoustic forcing amplitude might have a slightly higher maximum gain [19], it can be concluded that for the experimental conditions of this study, the flames are more sensitive to plasma forcing than to acoustic forcing. This result is of interest for practical applications of control of thermoacoustic instabilities by plasma actuator.

Figures 7 and 8 compare the transfer functions of the M-flame and the V-flame, respectively, obtained for the three types of forcing. To facilitate this comparison, the gains have been normalized to respective maximum. 

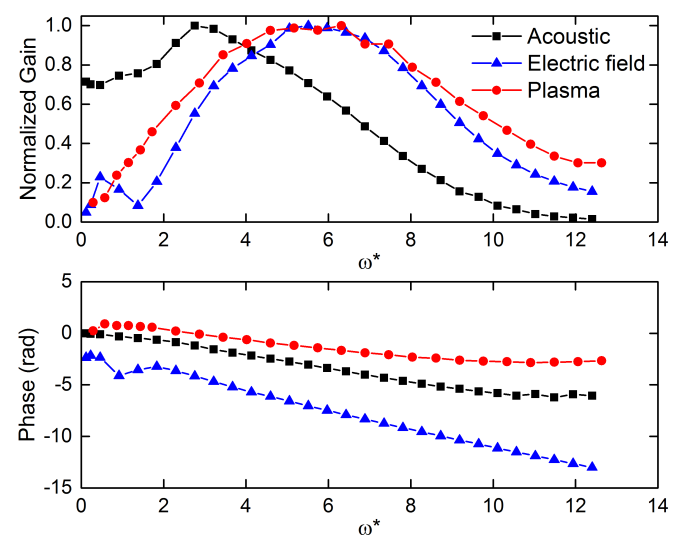

Figure 7: Comparison of the transfer functions of the M-flame to acoustic, AC electric field, and plasma forcing.
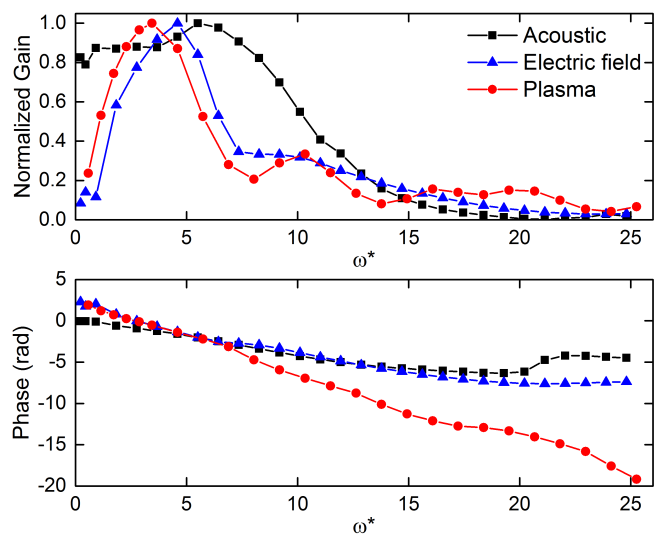

Figure 8: Comparison of the transfer functions of the V-flame to acoustic, AC electric field, and plasma forcing. 
For both M- and V-flames, the normalized gains of electric field and plasma forcing are very close, i.e. similar maximums and bandwidths. They differ from the gain of acoustic forcing by a shift of the maximums, to higher reduced frequencies for the M-flame and lower ones for the V-flame. It is interesting to note that for the two electrical forcing sources, M-flames are responsive over a larger range of frequencies. Finally, each type of forcing induces a different phase in the flame responses. Therefore it may be expected that different forcing mechanisms are responsible.

\section{Forcing mechanisms}

The acoustic forcing mechanisms are relatively well known in the case of laminar premixed C-, V-, and M-flames [16, 19]. Flames respond to the fluctuating velocity field through kinematic mechanisms. At low frequencies flame dynamics are dominated by a uniform motion, which evolves into a convected mode for higher frequencies. For example, the shapes of the phases, or the reduced cut-off frequencies of the gains, can reasonably be predicted by analytical kinematic models.

\subsection{Mechanism of forcing by AC electric field}

The electric field affects flame dynamics mainly by the acceleration of charged particles naturally present in the combustion zone (such as $\mathrm{H}_{3} \mathrm{O}^{+}$, $\mathrm{CHO}^{+}, \mathrm{C}_{3} \mathrm{H}_{3}^{+}, \mathrm{O}_{2}^{-}, \mathrm{O}^{-}, \mathrm{OH}^{-}$and electrons $\left.[27,28]\right)$. These charged particles are accelerated by the AC electric field, according to the Lorentz force. By transfer momentum to neutral particles, the ions generate a flow, also called ionic wind, and changes in the transport properties inside, and close to, the reaction zone. 
In this study, when exposed to AC electric field forcing, the flames oscillate, irrespectively of their shape. This behavior indicates modification in either the upstream flow field or in the flame propagation speed. Indeed, it has been reported that a sub-critical electric field might modify transport properties, generating thermo-diffusive instabilities and leading to changes in the global burning rate [29]. As no thermo-diffusive wrinkling has been observed on the flame surface, the main action of the AC electric field may therefore be attributed to the perturbation of upstream flow velocity, via the generation of ionic wind. Similar flow field modifications have been reported in Bunsen flames via particle image velocimetry measurements [10]. Taking into account the geometry of the electrodes (point-to-plane, see Fig.1a), the electric field is highly non-uniform. Consequently, a non-uniform modification of the upstream flow field, leading to local changes in the flame surface area and stretching, can be expected. This mechanism is likely to be responsible for the measured HRR fluctuations. The similarity of the FTFs obtained for the three flame shapes may be explained by the small size of the flame compared to the electric-field gradient.

Therefore, if the ionic wind, $u_{i o n i c}$, is the key parameter for $\mathrm{AC}$ electric field forcing, the FTF can be expressed as:

$$
F_{u_{\text {ionic }}}(\omega)=\frac{\hat{\dot{Q}}(\omega) / \bar{Q}}{\hat{u}_{\text {ionic }}(\omega) / \bar{u}} .
$$

The forcing amplitude can then be defined as the ratio of the ionic wind fluctuation amplitude divided by the mean flow velocity. This FTF definition allows comparisons with acoustic forcing, but velocity measurements in the flame area will be required. A significant effect of $\mathrm{AC}$ electric field forcing 
on the flame dynamics can thus be expected if the ionic wind generated is non-negligible, compared to the main flow of the burner.

\subsection{Mechanism of forcing by NRP plasma discharges}

During forcing by NRP glow discharges, the flames are affected mainly by chemical and thermal activation of the fluid in the plasma area. This can also be expressed as an increase of the reactivity of the fluid in the plasma area, leading to an increase in the burning velocity (see for example [30, 31]). Owing to the geometry of the setup and the flame shapes, the density of plasma is higher close to the rod (see Fig. 1). Hence, in terms of the flame front kinematics, this may be interpreted as a local increase in the burning velocity, which is known to lead to flame front perturbations in a similar manner as those from velocity disturbances [32]. Therefore, the forcing mechanism by plasma actuation may be attributed to a local increase of the burning velocity close to the rod, modulated at the forcing frequency.

Differences in the FTFs of the two flame shapes can be explained by a different distribution of the plasma in the flame front. Indeed, for the Mflame, the emission light from the plasma is more homogeneous, compared to the V-flame, where the plasma is confined close to the rod (see Fig.1, pink color in the flame, coming from $\mathrm{N}_{2}(\mathrm{C}-\mathrm{B})$ transition). This difference could change the burning rate gradient, and consequently should affect the response of the flame to plasma forcing, and the associated transfer function. Further investigation will be necessary to verify this explanation. 


\section{Conclusion}

The transfer functions of laminar methane-air flames for forcing by acoustic waves, AC electric fields, and nanosecond repetitively pulsed (NRP) glow discharges have been investigated and compared.

First, it has been shown that, in the range of 4 to $450 \mathrm{~Hz}$, plasma and electric field forcing can significantly affect the heat release rate of the C-, V-, and M-flames. In addition, it has been found that in the case of forcing by acoustic waves and plasma generated by NRP glow discharges, the geometry of the flame plays a key role on the response of the combustion, while the flame shape does not greatly affect the response of the combustion to AC electric field forcing.

Flame responses to acoustic forcing of about $10 \%$ amplitude of the incoming flow are similar to those obtained in previous studies, and significantly lower than the response of the flame to plasma forcing, for a forcing amplitude of $1.8 \%$.

A possible forcing mechanism for AC electric fields, through the modification of the upstream flow field via generation of ionic wind, has been proposed. For plasma forcing, a local increase in the burning velocity close to the rod, inducing wrinkling of the flame front, may explain the results. Further investigations will be necessary to verify the validity of these forcing mechanisms.

Finally, as the flame response to the three forcing types is very similar, this study reinforces the idea of controlling acoustically induced combustion instabilities with either AC electric field or non-thermal plasma. 


\section{Acknowledgements}

This work is partly funded by the Agence Nationale de la Recherche and the German Research Foundation, through the DRACO project (ANR-13IS09-0004), and by the Center Competitive Funding from King Abdullah University of Science and Technology (KAUST).

[1] T. C. Lieuwen, V. Yang (Eds.), Combustion Instabilities in Gas Turbine Engines, Vol. 210 of Progress in Astronautics and Aeronautics, AIAA, Inc., 2005.

[2] S. Candel, Proc. Combust. Inst. 29 (1) (2002) 1-28.

[3] D. Lacoste, D. Xu, J. Moeck, C. Laux, Proc. Combust. Inst. 34 (2) (2013) 3259-3266.

[4] J. Moeck, D. Lacoste, C. Laux, C. Paschereit, in: Proc. 51st AIAA ASM, 2013, paper no. AIAA 2013-0565.

[5] D. Lacoste, J. Moeck, D. Durox, C. Laux, T. Schuller, J. Eng. Gas Turbines Power 135 (2013) 101501.

[6] J. D'Entremont, R. Gejji, P. Venkatesh, S. Bane, in: Proc. 52nd AIAA ASM, 2014, paper no. AIAA 2014-0622.

[7] W. Kim, J. Snyder, J. Cohen, Proc. Combust. Inst. 35 (3) (2015) 34793486.

[8] D. Wisman, S. Marcum, B. Ganguly, Combust. Flame 151 (4) (2007) 639-648. 
[9] E. Volkov, V. Kornilov, L. de Goey, Proc. Combust. Inst. 34 (1) (2013) 955-962.

[10] J. Kuhl, G. Jovicic, L. Zigan, A. Leipertz, Proc. Combust. Inst. 34 (2) (2013) 3303-3310.

[11] J. Kuhl, G. Jovicic, L. Zigan, S. Will, A. Leipertz, Proc. Combust. Inst. 33 (3) (2015) 3521-3528.

[12] C. Guerra-Garcia, M. Martinez-Sanchez, Combust. Flame in press (2015) 10.

[13] Y. Xiong, D. Park, B. Lee, S. Chung, M. Cha, Combust. Flame in press (2015) 9.

[14] Y. Xiong, M. Cha, S. Chung, Proc. Combust. Inst. 35 (3) (2015) 35133520.

[15] A. Drews, L. Cademartiri, M. Chemama, M. Brenner, G. Whitesides, K. Bishop, Phys. Rev. E 86 (2012) 036314.

[16] T. Schuller, D. Durox, S. Candel, Combust. Flame 134 (1-2) (2003) 21-34.

[17] W. Polifke, C. Lawn, Combust. Flame 151 (3) (2007) 437-451.

[18] B. Bellows, M. Bobba, A. Forte, J. Seltzman, T. Lieuwen, Proc. Combust. Inst. 31 (2) (2007) 3181-3188.

[19] D. Durox, T. Schuller, N. Noiray, S. Candel, Proc. Combust. Inst. 32 (2009) 1391-1398. 
[20] K. Kim, J. Lee, B. Quay, D. Santavicca, Combust. Flame 157 (9) (2010) $1718-1730$.

[21] A. Mazas, B. Fiorina, D. Lacoste, T. Schuller, Combust. Flame 158 (12) (2011) 2428-2440.

[22] P. Palies, D. Durox, T. Schuller, S. Candel, Combust. Sci. and Tech. 183 (2011) 704-717.

[23] D. Rusterholtz, D. Lacoste, G. Stancu, D. Pai, C. Laux, J. Phys. D : Appl. Phys. 46 (2013) 464010 (21pp).

[24] D. Pai, G. Stancu, D. Lacoste, C. Laux, Plasma Sources Sci. Technol. 18 (4) (2009) 045030.

[25] I. Hurle, R. Price, T. Sugden, A. Thomas, Proc. R. Soc. London, Ser. A 303 (1475) (1968) 409-427.

[26] R. Price, I. Hurle, T. Sugden, Symp. (Int.) Combust. 12 (1) (1971) 10931102.

[27] A. Fialkov, Prog. Energy Combust. Sci. 23 (1997) 399-528.

[28] M. Belhi, P. Domingo, P. Vervisch, Combust. Theor. Model. 17 (4) (2013) $749-787$.

[29] S. Marcum, B. Ganguly, Combust. Flame 143 (1-2) (2005) 27-36.

[30] T. Ombrello, S. Won, Y. Ju, S. Williams, Combust. Flame 157 (2010) $1906-1915$.

[31] K. Zaima, K. Sasaki, Jap. J. Appl. Phys. 53 (6) (2014) 066202. 
[32] J. H. Cho, T. Lieuwen, Combust. Flame 140 (2005) 116-129. 


\section{Figure captions}

Figure 1: Experimental setup used for a) acoustic and $\mathrm{AC}$ electric field forcing with three flame geometries: conical (C), M-shaped (M), and Vshaped (V) flames, and b) plasma forcing by NRP glow discharges with two flame geometries: M-shaped (M) and V-shaped (V) flames.

Figure 2: a) Comparison of flame spectrum (blue), spectrum of flame with an AC electric field (red), and flame emission between two pulses of NRP glow discharges (black). b) Temporal evolution of the global $\mathrm{CH}^{*}$ emission of an M-flame between two pulses of plasma.

Figure 3: a) Example of forcing signals used for a forcing at $196 \mathrm{~Hz}$. b) Corresponding temporal evolution of the normalized $\mathrm{CH}^{*}$ emission of the M-flame.

Figure 4: C-flame, V-flame and M-flame transfer functions to acoustic forcing (forcing amplitude 10\%).

Figure 5: C-flame, V-flame and M-flame transfer functions to AC electric field forcing.

Figure 6: V-flame and M-flame transfer functions to non-thermal plasma generated by NRP glow discharges at $10 \mathrm{kHz}$ (forcing amplitude 1.8\%).

Figure 7: Comparison of the transfer functions of the M-flame to acoustic, $\mathrm{AC}$ electric field, and plasma forcing.

Figure 8: Comparison of the transfer functions of the V-flame to acoustic, $\mathrm{AC}$ electric field, and plasma forcing. 


\section{Supplemental material}

Figure S1: Movie of the flame motion induced by a 2-W plasma forcing at $64 \mathrm{~Hz}$, realized from $16 \mathrm{CH}^{*}$-chemiluminescence images, taken phase-locked with the forcing signal. 